\title{
Design of Intelligent supermarket cashier service system
}

\author{
Zhiyuan Chen ${ }^{1, a}$, Yameng Zhai ${ }^{2, b}$ and Li Tian ${ }^{1, c}$ \\ ${ }^{1}$ School of Mechanical Engineering, Dalian Jiaotong University, Dalian 116028, China; \\ ${ }^{2}$ School of Electronic Engineering, Dalian Jiaotong University, Dalian 116028, China. \\ a1003838876@qq.com, ${ }^{b} 13804250163 @ 163 . c o m,{ }^{c}$ xiiaotianli1993@163.com
}

Keywords: two-dimensional code identification, intelligent system, smart city.

\begin{abstract}
The intelligent cashier system uses LabVIEW to identify two-dimension codes and PLC to realize the movement control. The main functions of the system includes: transferring the stuffs based on the collection of location information by photoelectric sensor; taking photographs to identify the two-dimensional code during this time; calculating the amount by the internal systems; giving an shopping list based on database. Using a coin machine to collect coins, stepper motor to support the change back part, intelligent anti-theft to ensure a secure, accurate and comprehensive billing environment to meet all the needs of the shopping while at the same time saving energy and human resources.
\end{abstract}

\section{Introduction}

With the rapid development of social economy, the supermarkets have been played more and more important roles in daily life. However, the normal cashiers of supermarket relies on human to count and transfer the goods. This system will pass the goods and scanning to count the price automatically. Using this machine can avoid the mistakes made by human's careness, save resources and reduce time for customers ’waiting. It can provide customers a more intelligent living environment.

\section{System design}

The system is composed by six important parts, including the convey belt, the camera for scanning two-dimensional code and identifying the price of goods, the cash register, the coin returning subsystem and the intelligent guard against theft.

By using PLC to control the motor, coin count and intelligent anti-theft part, the system can realize all the function as designed. On both sides of the conveyor belt, there are three sets of photoelectric sensors, which can be used to detect the transmission position of the goods. The sensor which set in the middle of the conveyor belt can confirm whether the goods enter the film area or not, once it enter the scanning area, the system will turn on the camera to identify the $2 \mathrm{D}$ code, create a list of commodity information, and keep waiting for customers to pay the bill. The system uses an electromagnet as a switch to ensure the product which haven't been paid couldn't be taken away[1-3].

The function of change has been achieved by step motor, through the hardware system with the stepper motor the system can return the change of coin, the coin pop card will be prepared in advance. The step motor drives the coin pop card to rotate, and the number of the coin is determined according to the number of the input pulses. Because the two-dimensional code has many advantages, such as large data storage capacity, high confidentiality, high tracking performance, strong anti-damage and so on. This system choice the $2 \mathrm{D}$ code as the subject to recognize instead of the traditional bar code. In the experiment, researchers generated custom two-dimensional code which including some attribute information such as the recent changes in commodity prices. Database has been built to provide private custom shopping guide of different custom based on the shopping record. 


\section{Software design}

\subsection{Programming based on LabVIEW}

Programming on LabVIEW to realize the following function: user interface design, image processing, and calculate the total price change, Access database design and TCP communication with PLC. The function chart of LabVIEW has been shown in Fig. 1.

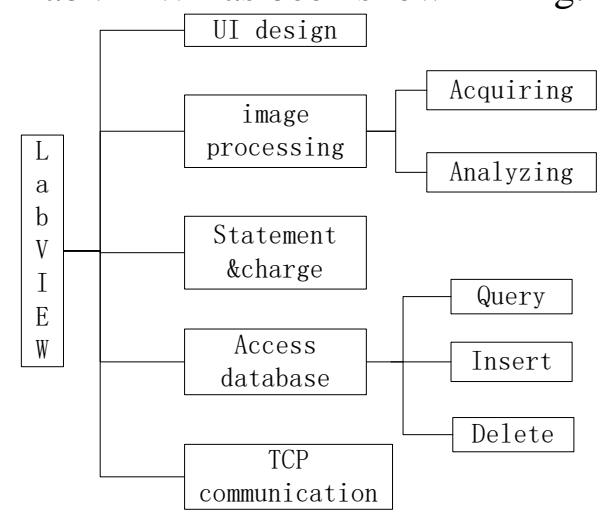

Fig. 1 The function chart of LabVIEW

Image process including image acquisition and image recognition (two-dimensional code recognition), Access database design contains the functions of the database query, add and delete. As shown in Fig. 2 is part of program of picture processing[4].

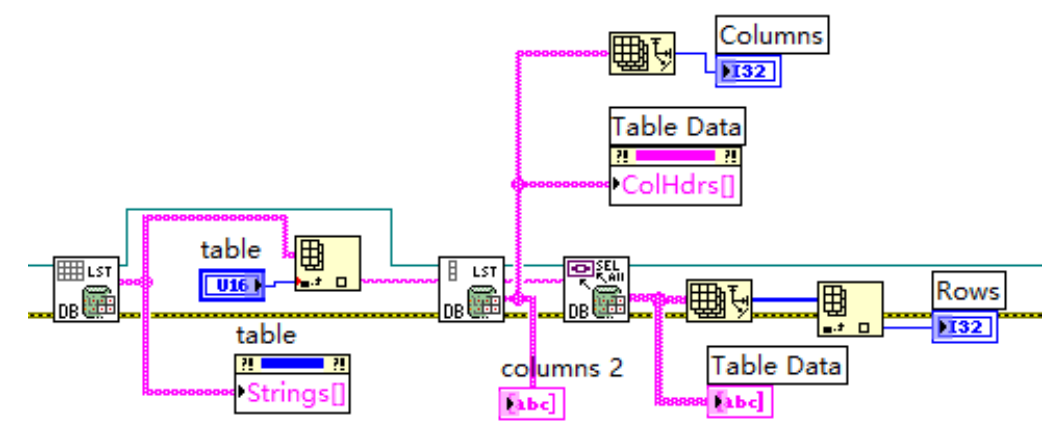

Fig. 2 Image acquisition and recognition

To realize the function of two-dimensional code identification, it is necessary to use the vision tool kit for image processing. Figure 2 shows the image recognition program. When the system starts the camera, the image stream will be storage in the queue. When the customer finished shopping, the program automatically calculates the sum of consumption, and gives a clear notice on the front panel. It is convenient for the user to pay the bill according to the actual price shown in the panel. After payment, the system will calculate the amount of coin the user actually inserts. Only the user pays successfully, anti-theft system will lift the restrictions, users can take goods away. The corresponding database will be created to record all the information of this shopping.

\subsection{Programming based on PLC}

The conveyor belt movement is controlled by the DC motor to drive the PLC.As is shown in Fig.3 is the function chart of PLC.

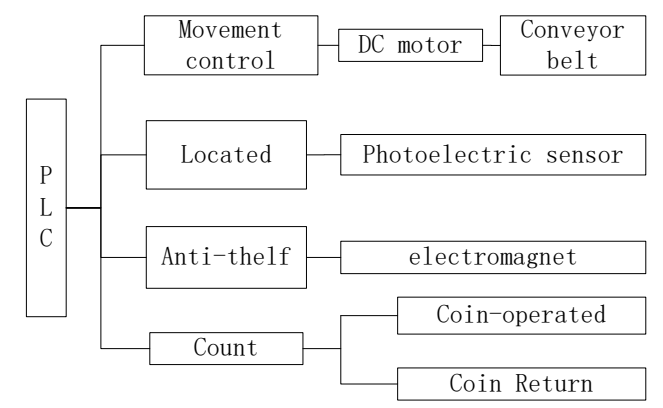

Fig. 3 Function chart off PLC 
When the goods pass the conveyor belt are arranged in the camera scan area for settlement in the region did not check, pick up the mouth door is closed, when the coin and the change after the closing, the electromagnet to open the door, then you can extract, when the sensor detects the goods are removed after the door closed again, the electromagnet reset. In practical application, complete the merchandise in this system is also degaussing.

Coin -operator is connect to the PLC through signal line, when coins come in, only after the coin recognition recognized as qualified the coin can pass through, then start counting and sent the number to the buffer area, each coin, count plus one the result is for the calculation and change ,it also shows on the display. Part of the program has been shown in Fig. 4.

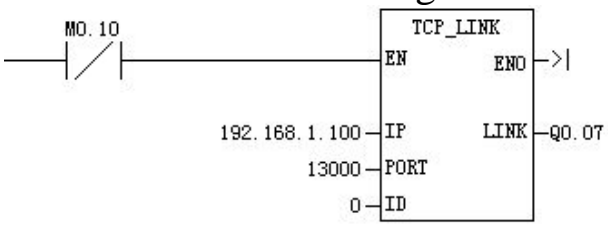

Fig. 4 Part of the PLC subsystem

The coin return system is consists of PLC and stepper motor, coin slot. When the customer clicks Change, the system calculates the change value, according to the number of change, send the corresponding number of pulses, stepper motor drive coin card slot rotation corresponding angle, return the coin out.

\subsection{TCP communication}

Because TCP communication can guarantee the upper computer and lower position machine communication in time, as well as the data accuracy is not lost. So choose TCP communication protocol. Figure 5 is a program block diagram of TCP communication between LabVIEW and PLC[5].

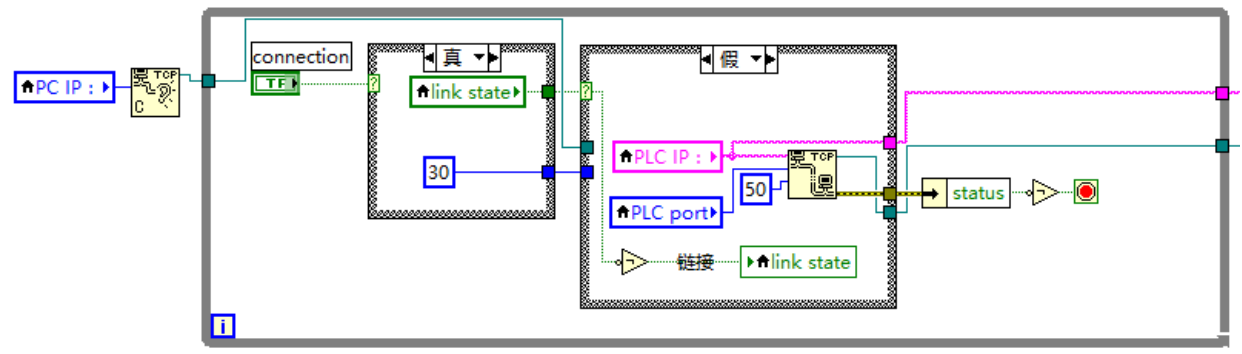

Fig. 5 Program block diagram of TCP communication

As shown in Figure 5, after setting the port number, start the program, the program will first enter the interception loop, only when the upper computer detects the presence of the lower computer , and successfully match, the program will continue to perform, the cashier to work properly.

\section{Experiment and test}

Start the program, when the identification of goods need to be sent and identified, the conveyor belt began to move. When the goods reach the camera can be taken within the scope of the camera, the camera, as shown in Fig. 6. 


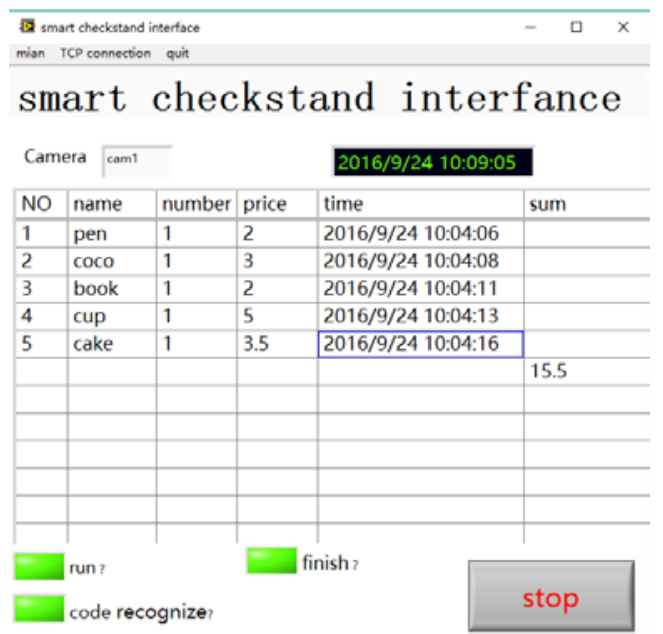

Fig. 6 The interface of the system

When it is confirmed that all the goods have been passed through the conveyor belt, the system will automatically calculate the total price of goods, and return to the main interface of the program. Insert coins according to the total price after the user checking the shopping information.

In order to ensure that each consume has a record, convenient the management of goods for the shopping mall, the program will automatically stored time and product information at the same time when it runs. Based on the information stored in the database, the system can analyzes and statistics, concluded the type of customer's favorite goods and other information, and give a recommendation of the shopping list as a reference for customers next time shopping.

\section{Conclusion}

Advantages of this system:

1. Recognizing QR code by LabVIEW. It will expand the identification code storage which contains products information and broaden shopping horizons.

2. Building a database to store shopping information. It can analyze data and design customized shopping list for different customers.

3. User Interface is simple and easy to operate. This system is caters for all ages. People can operate the system on their own.

4 This system contains change system which is made by us and intelligent anti-theft system. It will gather products which have passed the camera together. It is very convenient for customers to put things in bag. This system creates a safe and harmonious shopping environment.

With the development of the technology of Internet of things. Intelligent unmanned cash register process, can save time and energy. It also broadens shopping horizons, improve accuracy and safety. This system satisfies people's needs in this field, has a certain market prospects.

\section{References}

[1] Ltd. V. System and method for improved quality management in a product logistic chain[J].2016.

[2] Daboub J A, Rodrigues R. System and method for providing goods, services or information using scannable code[J]. 2016.

[3] SONG Kai, Kenfeng Seed CO. The Application of PLC in the Electrical Automatic Control[J]. Times Agricultural Machinery, 2016.

[4] Junchao L I, Ketian L I. A Detecting System of 2-Dimensional Bar Code Based on LabVIEW and Vision[J]. Machine Tool \& Hydraulics, 2011.

[5] Ding Y X. The recognition system of 2-D bar code based on LabVIEW[J]. Microcomputer Information, 2010. 